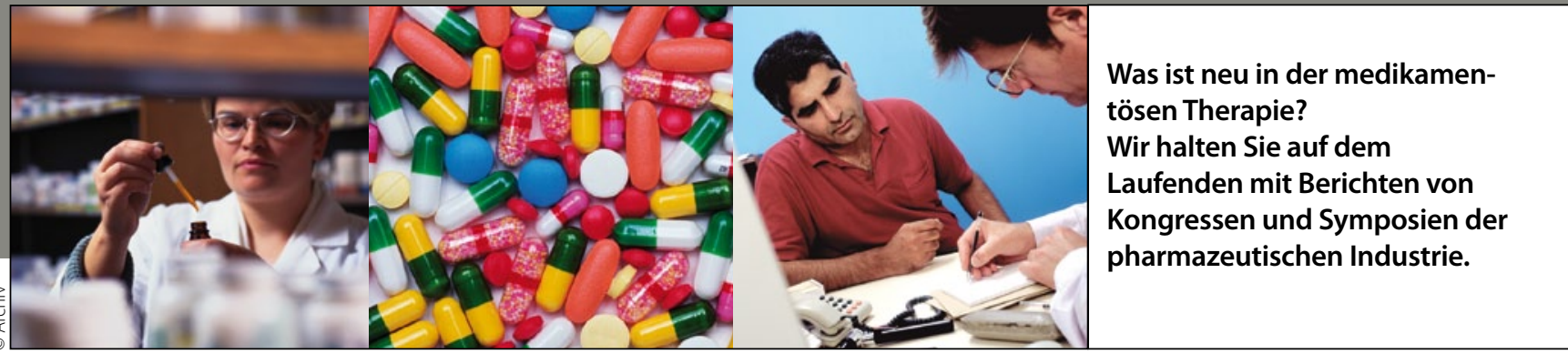

Asthma bronchiale

\title{
Neue Fixkombination vereinfacht und verbessert die Behandlung
}

- Die Einführung der Fixkombinationen mit einem Beta-2-Mimetikum und einem inhalierbaren Glukokortikoid hat die Therapie des Asthma bronchiale nicht nur vereinfacht, sondern auch wesentlich verbessert. „Der eigentliche Charme einer Fixkombination besteht darin, dass der $\mathrm{Pa}$ tient, sobald er ein Medikament für die Symptomkontrolle einnimmt, quasi gezwungen wird, gleichzeitig eine Substanz zu inhalieren, die den dem Asthma bronchiale zugrundeliegenden Entzündungsprozess günstig beeinflusst", sagte Prof. Christian Virchow, Rostock.

flutiform ${ }^{\circledast}$ enthält als selektiven Beta-2Rezeptoragonisten Formoterol, das durch einen sehr schnellen Wirkeintritt und eine lange Wirksamkeit charakterisiert ist. Als Kombinationspartner wurde das ebenfalls klinisch bewährte und hoch wirksame inhalierbare Glukokortikoid Fluticason gewählt, das eine hohe relative Rezeptoraffinität, eine lange Inhalationshalbwertzeit und eine geringe systemische Bioverfügbarkeit verspricht. „In entsprechenden klinischen Stu-

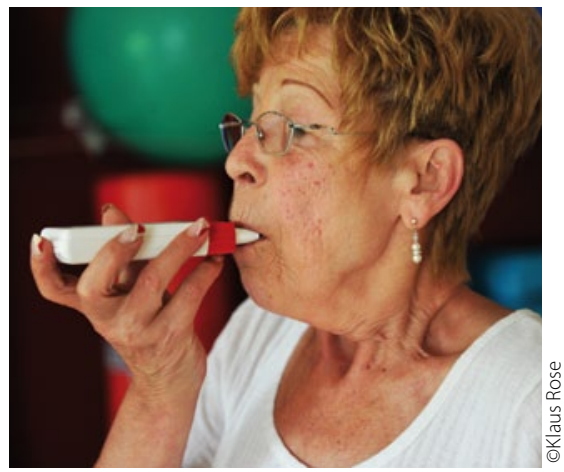

Mit der neuen Zweifach-Kombination kann sie ihr Asthma leichter kontrollieren. dien konnte überzeugend gezeigt werden, dass diese neue Fixkombination eine dauerhaft starke Wirkung bei schnellem Wirkeintritt und ein hohes Maß an Sicherheit und Verträglichkeit bietet", so Virchow.

\section{Wann ist die Fixkombi indiziert?}

Indiziert ist die Fixkombination bei Patienten, die mit inhalativen Kortikosteroiden und bedarfsweise angewendeten kurz wirksamen inhalativen Beta-2-Agonisten nicht ausreichend eingestellt sind bzw. bei Patienten, die mit einem inhalativen Kortikosteroid und einem lang wirksamen BetaAgonisten in Kombination eingestellt sind. Die Fixkombination steht in drei verschiedenen Wirkstärken zur Verfügung, was eine flexible Anpassung an den Schweregrad der Erkrankung und das individuelle Ansprechen des Patienten ermöglicht $(50 \mu \mathrm{g} / 5 \mu \mathrm{g}, 125 \mu \mathrm{g} / 5 \mu \mathrm{g}$ und $250 \mu \mathrm{g} / 10 \mu \mathrm{g}$ Fluticason/Formoterol).

Beim Inhalationssystem von flutiform ${ }^{\circledast}$ handelt es sich um ein den Patienten vertrautes, modernes Treibgas-betriebenes Dosieraerosol, das 120 Sprühstöße enthält. „Das Device in modernem Design verleiht dem Patienten Sicherheit bei der Planung dank eines leicht ablesbaren Dosiszählers", so Dr. Thomas Voshaar, Moers. Neben einer Farbkodierung nach dem Ampelsystem (grün-gelb-rot) besitzt das Zählwerk auch eine nummerische Anzeige der noch verbleibenden Sprühstöße.

\section{- Dr. med. Peter Stiefelhagen}

Quelle: Pressekonferenz „flutiform ${ }^{\oplus}$ : Neue Fixkombination zur Behandlung von Asthma bronchiale", ERS-Jahrestagung, Wien, September 2012 (Veranstalter: Mundipharma)
Kurz notiert

Smartphone-App hilft bei entzündlichen Darmerkrankungen Mit einer kostenlosen App (http:// mobile.falkfoundation.org/app) können Sie schnell und unkompliziert die Leitlinien-Empfehlungen für Morbus Crohn und Colitis ulcerosa abrufen nicht nur in der Praxis, sondern auch von unterwegs.

Falk Foundation

Medikamentös unterstützte Adipositastherapie $>$ Die Indikation für eine adjuvante medikamentöse Adipositastherapie kann bei Patienten mit einem $B M I \geq 30 \mathrm{~kg} / \mathrm{m}^{2}$ gegeben sein, die mit der Basistherapie (Ernährungsumstellung und mehr Bewegung) keinen ausreichenden Erfolg hatten sowie bei Patienten mit BMI $\geq$ $27 \mathrm{~kg} / \mathrm{m}^{2}$, bei denen neben einer erfolglosen Basistherapie gravierende Risikofaktoren und/oder Komorbiditäten bestehen. In Erwägung gezogen werden kann ein zentral wirksames Antiadipositum wie Alvalin ${ }^{\circledR}$, das verschreibungspflichtig ist.

Der enthaltene Wirkstoff Cathin gehört zur Gruppe der indirekten Sympathomimetika. Durch die Erhöhung der Neurotransmitterkonzentration im synaptischen Spalt der Nervenzellen im Sympathikus wird das Hungergefühl reduziert, das Schlafbedürfnis gesenkt und die Leistungsfähigkeit erhöht. Da von dem Wirkstoff ein Gewöhnungs- und Suchtpotenzial bekannt ist, sollte das Medikament nur im Rahmen einer multimodalen Therapie und unter ärztlicher Kontrolle nicht länger als vier bis sechs Wochen eingesetzt werden. Riemser 